\title{
Atractylenolide III Enhances Energy Metabolism by Increasing the SIRT-1 and PGC1 $a$ Expression with AMPK Phosphorylation in C2C12 Mouse Skeletal Muscle Cells
}

\author{
Mi Young Song, ${ }^{a, \#}$ Hyo Won Jung, ${ }^{b, c, \#}$ Seok Yong Kang, ${ }^{b, c}$ and Yong-Ki Park*,b,c \\ ${ }^{a}$ Department of Rehabilitation Medicine of Korean Medicine, Dongguk University; Dongdae-ro 123, Gyeongju 38066, \\ Republic of Korea: ${ }^{b}$ Department of Herbology, College of Korean Medicine, Dongguk University; Dongdae-ro 123, \\ Gyeongju 38066, Republic of Korea: and ${ }^{c}$ Korean Medicine R\&D Center, Dongguk University; Dongdae-ro 123, \\ Gyeongju 38066, Republic of Korea. \\ Received October 31, 2016; accepted December 10, 2016
}

Targeting energy expenditure provides a potential alternative strategy for achieving energy balance to combat obesity and the development of type 2 diabetes mellitus (T2DM). In the present study, we investigated whether atractylenolide III (AIII) regulates energy metabolism in skeletal muscle cells. Differentiated C2C12 myotubes were treated with AIII $(10,20$, or $50 \mu \mathrm{M})$ or metformin $(2.5 \mathrm{~mm})$ for indicated times. The levels of glucose uptake, the expressions of key mitochondrial biogenesis-related factors and their target genes were measured in $\mathrm{C} 2 \mathrm{C} 12$ myotubes. AIII significantly increased the glucose uptake levels, and significantly increased the expressions of peroxisome proliferator-activated receptor coactivator-1 $\alpha$ (PGC1 $\alpha)$ and mitochondrial biogenesis-related markers, such as, nuclear respiratory factor-1 (NRF-1), and mitochondrial transcription factor A (TFAM) and mitochondrial mass and total ATP contents. In addition, AIII significantly increased the phosphorylation of AMP-activated protein kinase (AMPK) and the expression of sirtuin1 (SIRT1). These results suggest that AIII may have beneficial effects on obesity and T2DM by improving energy metabolism in skeletal muscle.

Key words atractylenolide III; mitochondrial biogenesis; AMP-activated protein kinase; peroxisome proliferator-activated receptor coactivator- $1 \alpha$; sirtuin1; C2C12 cell

The prevalence of obesity, metabolic syndrome, and type 2 diabetes mellitus (T2DM) has increased over past decades, ${ }^{1)}$ and it is estimated over 1.9 billion adults worldwide are overweight, and that more than 600 million are clinically obese. ${ }^{2)}$ Obesity, T2DM, and metabolic syndrome are among the most frequent pathological consequences of chronic energy metabolism imbalance. ${ }^{3)}$ The maintenance of energy balance depends on the regulations of energy intake and expenditure. However, the long-term efficacies of reduced energy intake, such as, by calorific restriction, are problematic, and thus, the targeting of energy expenditure could provide a more effective means of promoting energy balance and combating obesity and T2DM. ${ }^{4}$

Skeletal muscle is regarded a target organ in the context of cellular bioenergetics, and is known to play an important role in the maintenance of glucose homeostasis and insulin sensitivity. Insulin resistance is associated with myocellular lipid accumulation, and it has been shown that insulin resistance is caused or accelerated by impaired oxidative capacity of skeletal muscle. ${ }^{5,6)}$ One study conducted under euglycemic conditions showed that skeletal muscle is responsible for $c a$. $80 \%$ of total body glucose uptake. ${ }^{7)}$

Peroxisome proliferator-activated receptor coactivator $1 \alpha$ (PGC1 $\alpha$ ), AMP-activated protein kinase (AMPK) and sirtuin1 (SIRT1) compose an energy sensing network that controls energy expenditure in skeletal muscle. ${ }^{8)} \mathrm{PGC} 1 \alpha$ plays a central role in the regulation of cellular energy metabolism, which results from the up-regulation of oxidative metabolism and the stimulation of mitochondrial biogenesis. ${ }^{9)}$ Furthermore, it is known metabolic sensors, such as AMPK, SIRT1 and PGC1 $\alpha$,

\# These authors contributed equally to this work. constitute vital links in the network responsible for the regulation of cellular energy metabolism. ${ }^{8)}$

In recent years, a number of natural compounds have been shown to increase energy expenditure in skeletal muscle by increasing mitochondrial biogenesis and oxidative phosphorylation via the activations of $\mathrm{PGC} 1 \alpha$, AMPK, and SIRT1. ${ }^{10,11)}$ And, in a previous study, we found the root extract of Atractylodes macrocephala KoIDzUmi (Atractylodis Rhizoma Alba; ARA) increases glucose and lipid metabolism in skeletal muscle by enhancing the activities of PGC1 $\alpha$, AMPK and SIRT1. ${ }^{12)}$ Atractylenolide III (AIII) is the primary bioactive compound of this extract. ${ }^{13)}$ AIII has been previously reported to have anti-inflammatory ${ }^{14,15)}$ and gastroprotective effects, ${ }^{16}$ ) however its effects on skeletal muscle energy metabolism have not been investigated. Therefore, in the present study, we investigated the effects of AIII on energy metabolism via PGC1 $\alpha /$ SIRT1/AMPK pathway activation in mouse skeletal muscle cells.

\section{MATERIALS AND METHODS}

Cell Culture and Treatments Mouse $\mathrm{C} 2 \mathrm{C} 12$ myoblasts (CRL-1772: Manassas, VA, U.S.A.) maintained in Dulbecco's Modified Eagle's Medium (DMEM, GibcoBRL, U.S.A.) supplemented with $10 \%$ fetal bovine serum (FBS) and a penicillin/streptomycin mix (Invitrogen, Grand Island, NY, U.S.A.) were used in the study. When cells were confluent, they were induced to differentiate into myotubes by placing them in differentiation medium (DMEM supplemented with 2\% horse serum; Invitrogen) for $4 \mathrm{~d}$. $\mathrm{C} 2 \mathrm{C} 12$ myotubes were then treated with or without different concentrations of AIII (A2987, 
Sigma-Aldrich, St. Louis, MO, U.S.A.) at 10, 20 and $50 \mu \mathrm{M}$ or metformin (the reference drug) at $2.5 \mathrm{~mm}$.

Glucose Uptake Assay The amount of glucose uptake in $\mathrm{C} 2 \mathrm{C} 12$ myotubes were measured using Glucose uptake cell-based assay kit (Cayman Chemical Co., Ann Arbor, MI, U.S.A.). Briefly, $\mathrm{C} 2 \mathrm{C} 12$ myotubes were placed in $1 \mathrm{~mL}$ of glucose-free medium containing $150 \mu \mathrm{g} / \mathrm{mL}$ of 2-[N-(7-nitrobenz-2-oxa-1,3-diazol-4-yl)amino]-2-deoxy-D-glucose (NBDG) the wells of a 4 -well plate for $4 \mathrm{~h}$. Supernatants were then removed and $200 \mu \mathrm{L}$ of cell-based assay buffer was added to each well. Amounts of 2-NBDG taken up by cells were determined by fluorescence microscopy (Leica Biosystems, Wetzlar, Germany).

Western Blot Cells were lysed in ice-cold lysis buffer $(0.1 \mathrm{~mL}$ of $50 \mathrm{~mm}$ Tris- $\mathrm{HCl}(\mathrm{pH} 7.2)$ containing $1 \%$ sodium deoxycholate, $0.1 \%$ sodium dodecyl sulfate (SDS), $0.15 \mathrm{M} \mathrm{NaCl}$, and $1 \%$ NP-40), and lysates were centrifuged at $12000 \times \boldsymbol{g}$ for $20 \mathrm{~min}$ at $4^{\circ} \mathrm{C}$. After measuring protein content using a bicinchoninic acid (BCA) assay, equal amounts of protein $(20 \mathrm{~g} / \mathrm{mL})$ were electrophoresed on $10 \%$ SDS-acrylamide gels, and then transferred to nitrocellulose membranes using an electrical transfer system. Non-specific binding was blocked by treating membranes with $3 \%$ skim milk in TBST buffer $(5 \mathrm{~mm}$ Tris- $\mathrm{HCl}, \mathrm{pH} 7.6,136 \mathrm{~mm} \mathrm{NaCl}$, and $0.1 \%$ Tween-20) for $1 \mathrm{~h}$. Blots were incubated for $1 \mathrm{~h}$ at room temperature (RT) with primary antibodies against anti-PGCl $\alpha$, anti-mitochondrial transcription factor A (TFAM), anti-nuclear respiratory factor-1 (NRF-1), anti-phospho-AMPK $\alpha$ (Thr 172), anti-AMPK $\alpha$, anti-SIRT1 (Cell Signaling Technology, Danvers, MA, U.S.A.), and anti- $\beta$-Actin (Sigma-Aldrich), incubated for $1 \mathrm{~h}$ at RT with horseradish peroxidase (HRP)-labeled anti-mouse immunoglobulin G (IgG) $(1: 1000$, Santa Cruz Biotechnology, MN, U.S.A.), washed three times with $1 \times$ TBST, and developed using ECL Western detection reagents (GE Healthcare BioSciences, PA, U.S.A.). Protein bands were quantified by densitometry using Image J software.

RT-PCR Total RNA was isolated from cells using TRIzol reagent, according to the manufacturer's instructions (GibcoBRL Life Technologies Inc., Grand Island, NY, U.S.A.), and quantified using a NanoDrop ND-1000 spectrophotometer (NadroDrop Technologies, Inc., Wilmington, DE, U.S.A.). cDNA was generated from $2 \mu \mathrm{g}$ of total RNA using a Reverse Transcription System kit (Promega, Fitchburg, WI, U.S.A.).
PCR was conducted using a Blend Taq PCR kit (Toyobo, Osaka, Japan) using the following conditions; $2 \mathrm{~min}$ at $94^{\circ} \mathrm{C}$ (pre-denaturation), $30 \mathrm{~s}$ at $94^{\circ} \mathrm{C}$ (denaturation), $30 \mathrm{~s}$ at $60^{\circ} \mathrm{C}$ (annealing), and $1 \mathrm{~min}$ at $72^{\circ} \mathrm{C}$ (extension) for 30 cycles. The following primers were used. $\mathrm{PGCl} \alpha$ : Forward; 5'-CAC CAA ACC CAC AGA AAA CAG-3', Reverse; 5'-GGGTCA GAG GAA GAG ATA AAGTTG, NRF-1 : Forward; 5'-AATGTC CGC AGT GAT GTCC-3', Reverse; 5'-GCC TGA GTT TGT GTT TGC TG-3', TFAM : Forward; 5'-CAC CCA GAT GCAAAA CTT TCA G-3', Reverse; 5'-CTGCTC TTT ATA CTT GCT CAC AG-3', SIRT1 : Forward; 5'-GATCCT TCA GTG TCA TGG TT-3', Reverse; 5'-GAA GACAATCTC TGGCTTCA-3', and glyceraldehyde-3-phosphate dehydrogenase (GAPDH): Forward 5'-GACATC ATA CTT GGCAGG-3', Reverse; 5'-CTC GTGGAG TCT ACT GGT-3'ACTGGT-3' used as the internal control. The bands were detected by UV and quantified by densitometry using Image J software (v. 1.48, NIH, U.S.A.)

Mitochondrial Mass Assay Mitochondrial mass was evaluated using 10- $N$-Nonyl acridine orange (NAO; a fluorescent probe) (ENZO Life Sciences, NY, U.S.A.). Briefly, C2C12 cells were incubated in DMEM containing $10 \mathrm{nM}$ of NAO for $1 \mathrm{~h}$ at $37^{\circ} \mathrm{C}$ in the dark, trypsinized, and resuspended in DMEM without NAO. Fluorescence intensities were measured using a luminometer (Promega) using excitation and emission wavelengths of 495 and $519 \mathrm{~nm}$, respectively.

ATP Content Assay Total ATP contents were determined using an ATP calorimetric assay kit (BioVision, Inc., Headquarters, CA, U.S.A.). Absorbance was measured at $570 \mathrm{~nm}$.

Data Analysis All experiments were performed in triplicate. Results are expressed as mean \pm standard errors of mean (S.E.M.). Statistical significance was determined by ANOVA followed by Tukey's test in GraphPad Prism (GraphPad Software, Inc., San Diego, CA, U.S.A.). Statistical significance was accepted for $p$ values $<0.05$.

\section{RESULTS}

Effect of AIII on Glucose Uptake in C2C12 Myotubes The glucose uptake assay showed that AIII at 20 or $50 \mu \mathrm{M}$ significantly increased myotube glucose uptake as compared with untreated control or cells treated with apigenin $(100 \mu \mathrm{m}$; a glucose transporter inhibitor) (Fig. 1). Using an 3-(4,5-dimethylthiazol-2-yl)-2,5-diphenyltetrazolium bromide (MTT) cell
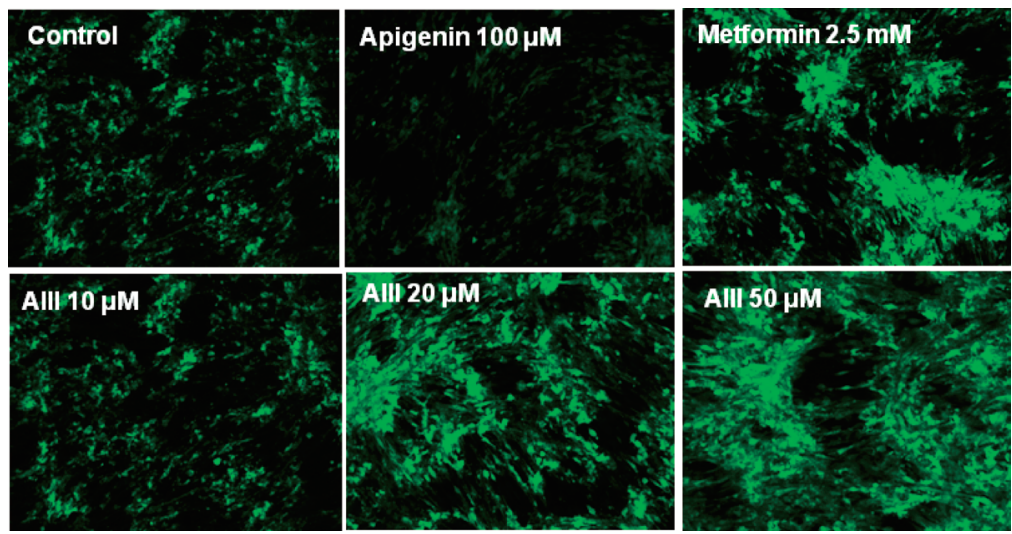

Fig. 1. Effect of AIII on Glucose Uptake in C2C12 Myotubes

Cells were treated with AIII, metformin $(2.5 \mathrm{~mm})$, or apigenin $(100 \mu \mathrm{M})$ for $4 \mathrm{~h}$. Glucose uptakes were observed by fluorescence microscopy after staining with 2-NBDG $(\times 100$ original magnification). 
A)

\begin{tabular}{l|ccccc} 
Alll $(\mu \mathrm{M})$ & 0 & 10 & 20 & 50 & 0 \\
Metformin $(\mathrm{mM})$ & 0 & 0 & 0 & 0 & 2.5 \\
PGC-1a & & & & &
\end{tabular}

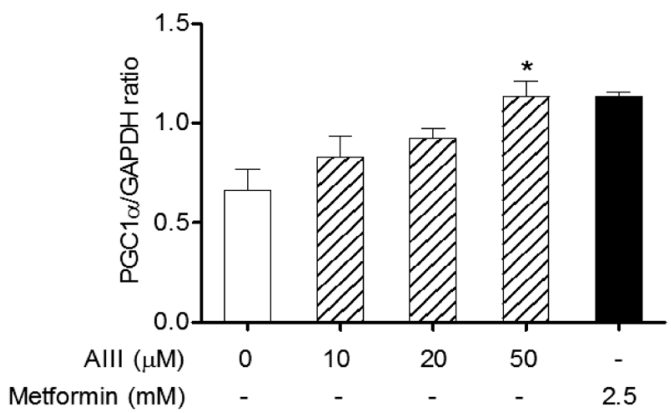

B)
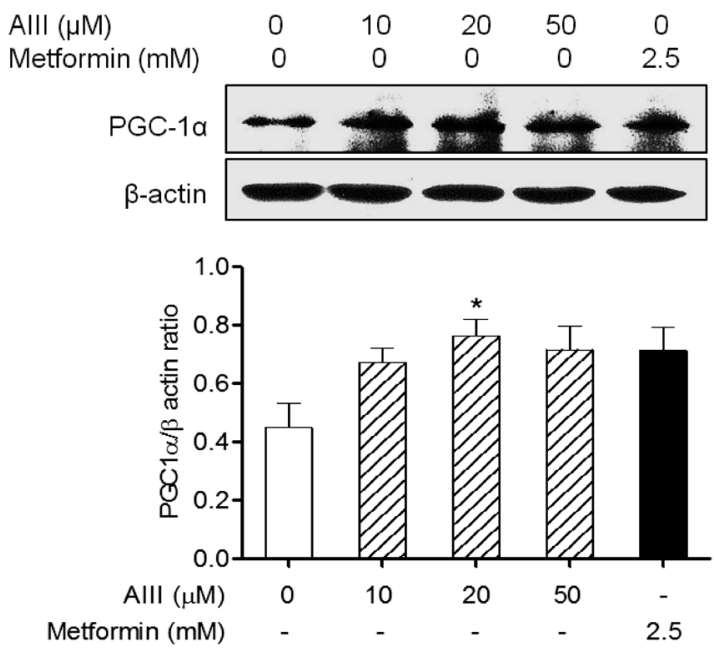

Fig. 2. Effect of AIII on PGC1 $\alpha$ Expression in $\mathrm{C} 2 \mathrm{C} 12$ Myotubes

Cells were treated with AIII (10, 20, or $50 \mu \mathrm{M}$ ) or ( $2.5 \mathrm{~mm}$ ) for $24 \mathrm{~h}$ (for mRNA) or $45 \mathrm{~min}$ (for protein). (A) PGC1 $\alpha$ mRNA levels were assessed by RT-PCR. (B) PGC1 $\alpha$ protein levels were assessed by Western blotting. Results are presented as mean \pm S.E.M. of three independent experiments. $* p<0.05 v s$. untreated cells.

A)

\begin{tabular}{|c|c|c|c|c|c|}
\hline $\begin{array}{l}\text { Alll }(\mu \mathrm{M}) \\
\text { Metformin }(\mathrm{mM})\end{array}$ & $\begin{array}{l}0 \\
0\end{array}$ & $\begin{array}{c}10 \\
0\end{array}$ & $\begin{array}{c}20 \\
0\end{array}$ & $\begin{array}{c}50 \\
0\end{array}$ & $\begin{array}{c}0 \\
25\end{array}$ \\
\hline NRF1 & $=$ & 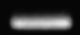 & 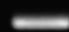 & 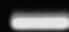 & $\Longrightarrow$ \\
\hline TFAM & $=$ & - & - & - & $=$ \\
\hline GAPDH & 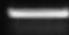 & - & & & \\
\hline
\end{tabular}

B)

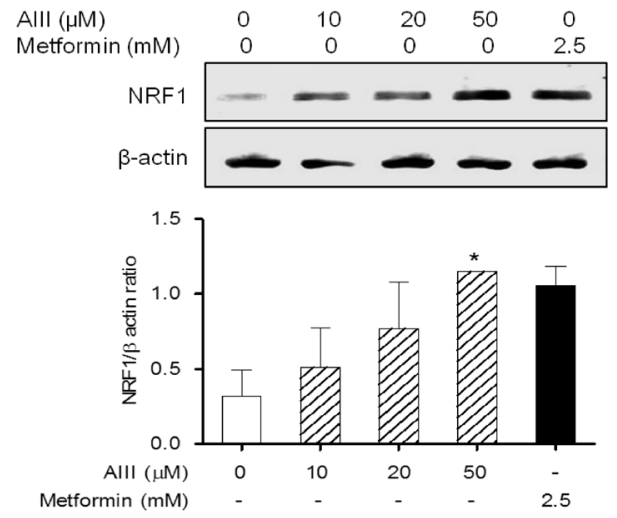

D)

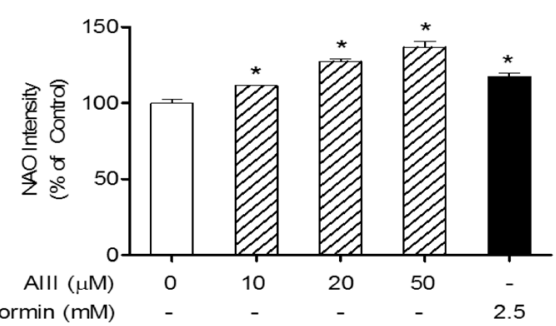

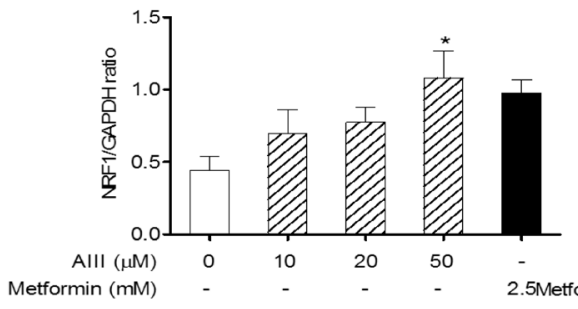

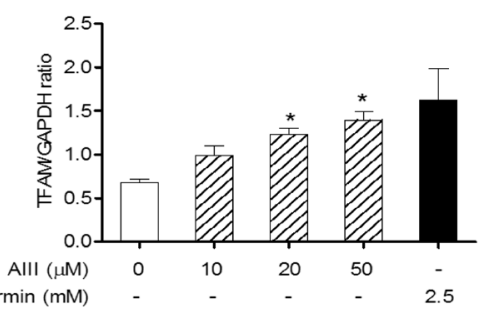

C)

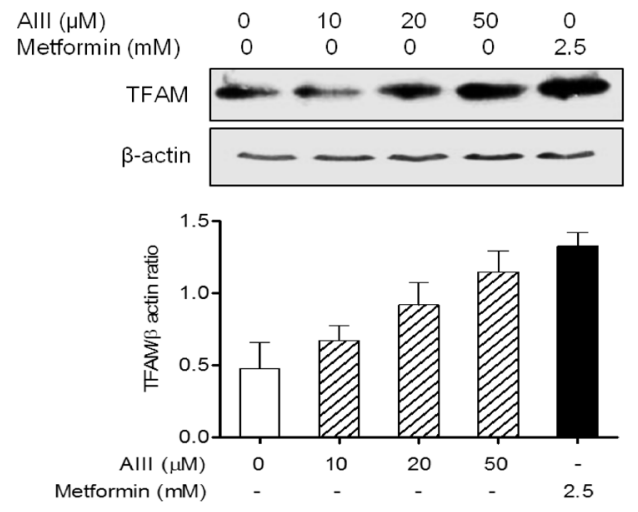

E)

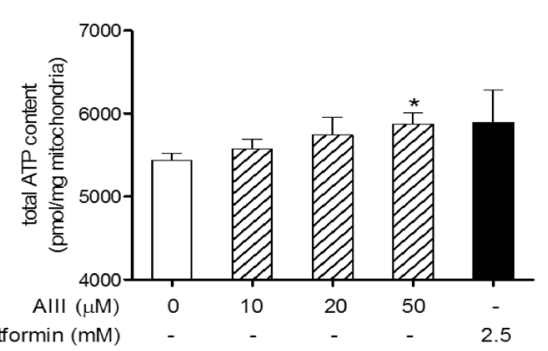

Fig. 3. Effect of AIII on Mitochondrial Biogenesis in $\mathrm{C} 2 \mathrm{C} 12$ Myotubes

Cells were treated with AIII (10, 20, or $50 \mu \mathrm{m})$ or $(2.5 \mathrm{~mm}$ ) for $24 \mathrm{~h}$ (for mRNA) or $45 \mathrm{~min}$ (for protein). (A) The mRNA expressions of NRF and TFAM were assessed by RT-PCR. (B and C) The protein expressions of NRF and TFAM were analyzed by Western blotting. (D) Mitochondrial mass and (E) total ATP content were measured by fluorescence staining with NAO or using an ATP assay, respectively. Results are presented as mean \pm S.E.M. of three independent experiments. $* p<.05 v s$. untreated cells. 
viability assay, AIII at 20 and $50 \mu \mathrm{M}$ have no toxicity in the cells (data not shown). Meanwhile, treatment with metformin also increased glucose uptake levels.

Effect of AIII on PGC1 $\alpha$ Expression in C2C12 Myotubes $\mathrm{PGCl} \alpha$ is a critical regulator of mitochondrial biogenesis and glucose metabolism in skeletal muscle and promotes response to exercise to maintain the balance between energy requirements and supply, ${ }^{9}$ ) and thus, we investigated the effects of AIII on PGC1 $\alpha$ expression in $\mathrm{C} 2 \mathrm{C} 12$ myotubes by RT-PCR and Western blotting. The expression of PGCl $\alpha$ mRNA (Fig. 2A) and protein (Fig. 2B) was increased in $\mathrm{C} 2 \mathrm{C} 12$ myotubes treated with AIII, indicating AIII enhances PGC1 $\alpha$ activity in skeletal muscle cells.

Effect of AIII on Mitochondrial Biogenesis in C2C12 Myotubes NRF-1 and TFAM are transcription factors targeted by PGC- $1 \alpha$ for the induction of mitochondrial biogenesis. ${ }^{17)}$ Thus, to determine whether AIII increases mitochondrial biogenesis in skeletal muscle, we examined the expressions of NRF-1 and TFAM in C2C12 myotubes by PCR and Western blotting, respectively. Treating $\mathrm{C} 2 \mathrm{C} 12$ myotubes with AIII dose-dependently increased the expressions of NRF-1 and TFAM at the mRNA (Fig. 3A) and protein levels (Figs. 3B, C), and similar increases were observed in metformin-treated cells.

Mitochondria are responsible for producing most of the ATP needed for energy-requiring reactions in eukaryotic cells, and ATP synthesis occurs in concert with expressional increases of key regulators of mitochondrial biogenesis. ${ }^{18)}$ Therefore, we measured the effect of AIII on ATP levels and mitochondrial mass using an ATP assay and by staining with $\mathrm{NAO}$, which binds to cardiolipin (a phospholipid specifically present on the mitochondrial membranes), ${ }^{19)}$ respectively. AIII dose-dependently increased myotube NAO fluorescence intensities (Fig. 3D) and total ATP contents (Fig. 3E), and similar results were observed in metformin-treated cells. These results indicate AIII increases mitochondrial biogenesis in skeletal muscle cells.

Effect of AIII on the Phosphorylation of AMPK in C2C12 Myotubes AMPK is a master sensor and regulator of

A)
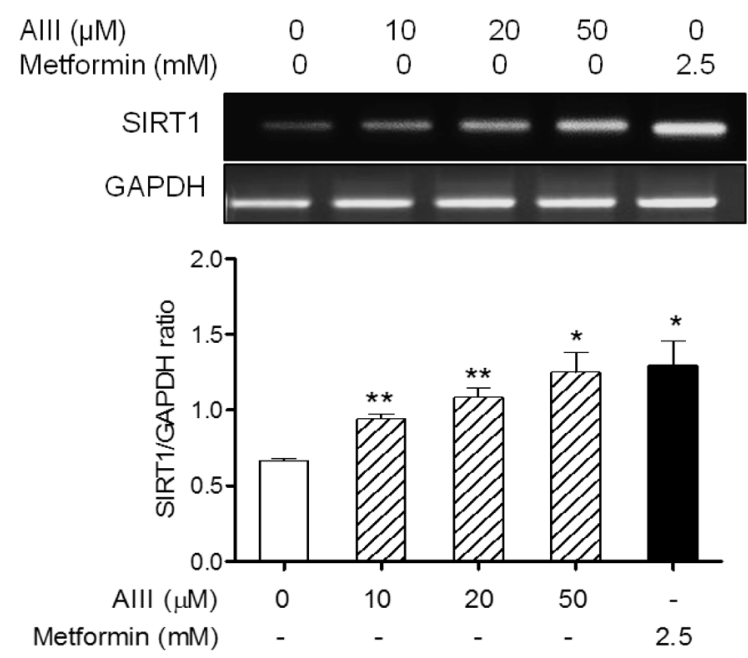

energy homeostasis at the cellular level, and plays fundamental roles in the regulations of glucose and lipid metabolism in skeletal muscle. ${ }^{20)}$ In addition, AMPK is also required for mitochondrial biogenesis and for the expressional up-regulation of PGC1 $\alpha$ in muscle. ${ }^{21)}$ Therefore, we investigated whether AIII regulates AMPK activity in $\mathrm{C} 2 \mathrm{C} 12$ myotubes by Western blot. AIII at 20 and $50 \mu \mathrm{M}$ was found to increase AMPK phosphorylation in $\mathrm{C} 2 \mathrm{C} 12$ myotubes versus untreated controls (Fig. 4). These results indicate that AIII increases glucose metabolism by activating the AMPK pathway in skeletal muscle cells.

Effect of AIII on SIRT1 Expression in C2C12 Myotubes AMPK enhances SIRT1 activity by increasing cellular oxidized form of nicotinamide adenine dinucleotide $\left(\mathrm{NAD}^{+}\right.$levels, which results in the deacetylations and modulations of the activities of downstream SIRT1 targets. Furthermore, this ex-

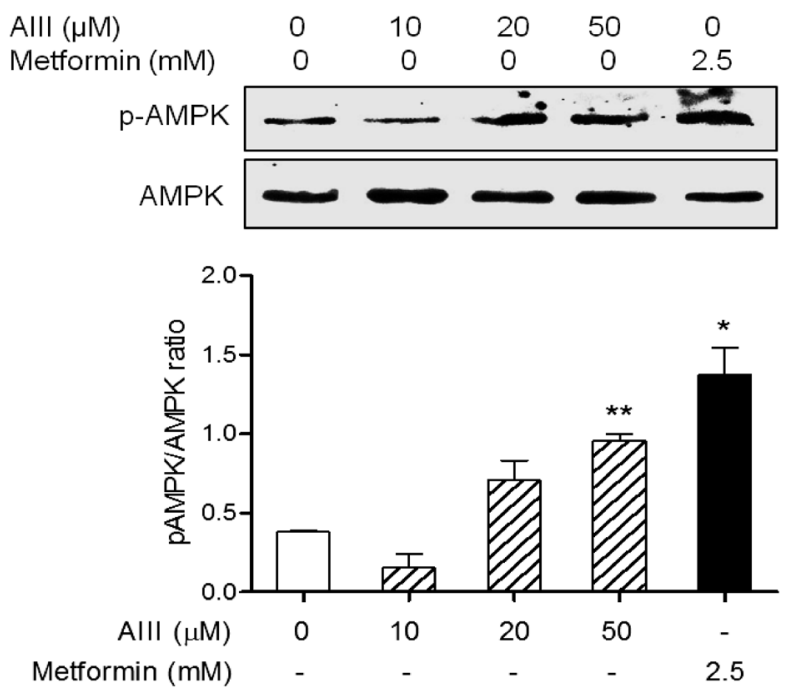

Fig. 4. Effect of AIII on the Phosphorylation of AMPK in C2C12 Myotubes

Cells were treated with AIII $(10,20$, and $50 \mu \mathrm{M})$ or metformin $(2.5 \mathrm{~mm})$ for $45 \mathrm{~min}$. The phosphorylation of AMPK was determined by Western blot, and histogram was presented as phospho- to total AMPK ratios. Results are presented as mean \pm S.E.M. of three independent experiments. ${ }^{*} p<0.05$ and $* * p<0.01 v s$. untreated cells.

B)

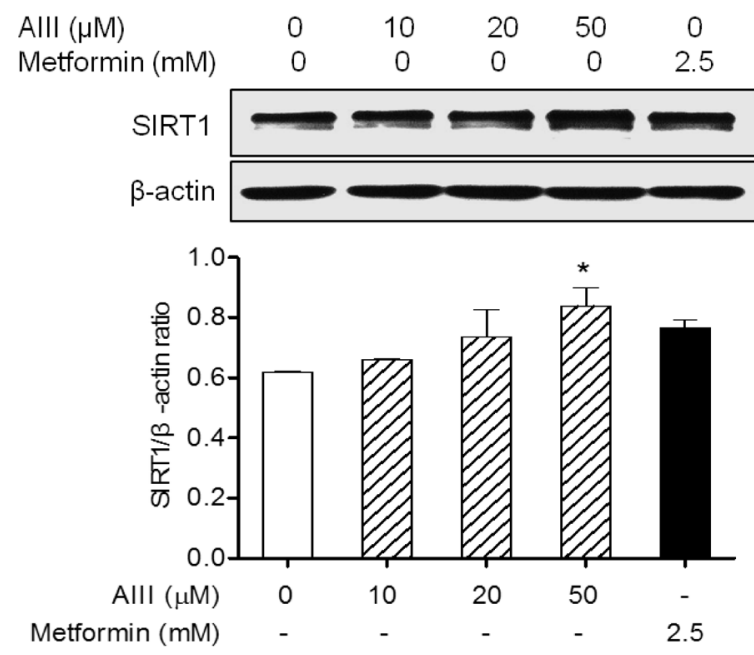

Fig. 5. Effect of AIII on the Expression of SIRT1 in C2C12 Myotubes

$\mathrm{C} 2 \mathrm{C} 12$ myotubes were treated with AIII $(10,20$, or $50 \mu \mathrm{M})$ or metformin $(2.5 \mathrm{~mm})$ for $24 \mathrm{~h}$. The expressions of SIRT1 at the mRNA (A) and protein (B) levels were analyzed by RT-PCR and Western blotting, respectively. Data was presented as mean \pm S.E.M. of three independent experiments. $* p<0.05$ and $* * p<0.01 v s$. untreated cells. 
plains many of the convergent biological effects of AMPK and SIRT1 in the context of energy metabolism. ${ }^{22}$ To investigate the effects of AIII on SIRT1 activity, we used RT-PCR and Western blotting to assess SIRT1 mRNA and protein levels. Treatment of $\mathrm{C} 2 \mathrm{C} 12$ myotubes with AIII was found to dosedependently increase SIRT1 mRNA (Fig. 5A) and protein (Fig. 5B) levels. These results indicate that AIII upregulates AMPK activation-mediated SIRT1 activity in skeletal muscle cells.

\section{DISCUSSION}

We previously reported that ARA extract increases glucose and lipid metabolism in $\mathrm{C} 2 \mathrm{C} 12$ myotubes by enhancing the activities of PGC1 $\alpha$, SIRT1, and AMPK. ${ }^{12)}$ This study was undertaken to determine whether AIII affects skeletal muscle cell energy metabolism. ARA actually contains three types of atractylenolides (I, II, and III), ${ }^{23}$ although AIII is the most abundant. ${ }^{13)}$ AIII has been reported to possess various biological activities, which include the inhibition of lipopolysaccharide-induced inflammatory responses in macrophages, ${ }^{14)}$ the suppression of histamine release from mast cells via the regulation of interleukin (IL)-6 at the cellular level, ${ }^{24)}$ and the induction of lung carcinoma cell apoptosis via cell growth inhibition and increasing lactate dehydrogenase release. ${ }^{15)}$ Here, we describe for the first time the regulatory effects of AIII on glucose homeostasis and energy metabolism in skeletal muscle cells.

The PGC1 $\alpha /$ SIRT1/AMPK pathway plays important roles in mitochondrial biogenesis and glucose metabolism in skeletal muscle. ${ }^{8,22,25)}$ In the present study, we found that AIII enhanced mitochondrial energy levels in $\mathrm{C} 2 \mathrm{C} 12$ myotubes and their glucose uptakes by activating this pathway. It has been shown PGC1 $\alpha$ increases energy expenditure by increasing mitochondrial biogenesis and respiration rates in skeletal muscle. $\left.{ }^{9}\right) \mathrm{PGC} 1 \alpha$ also interacts with NRF-1, a transcription factor of many mitochondrial genes, including TFAM a direct regulator of mitochondrial DNA replication and transcription. ${ }^{26}$ ) In the present study, the mRNA and protein levels of PGCl $\alpha$, NRF-1 and TFAM were dose-dependently increased by AIII treatment, and AIII significantly increased mitochondrial mass and total ATP content in $\mathrm{C} 2 \mathrm{C} 12$ myotubes. These results indicate that AIII can increase mitochondrial biogenesis by activating PGC1 $\alpha, \mathrm{NRF}-1$, and TFAM, in skeletal muscle cells.

AMPK and SIRT1 play major roles in the metabolic regulation of skeletal muscle, and have been reported to impact PGC1 $\alpha$ expression, and thus, to transcriptionally regulate energy expenditure. ${ }^{8)}$ AMPK switches on catabolic processes that provide alternative routes for ATP generation, glucose uptake, glycolysis, fatty acid oxidation, and mitochondrial biogenesis, but switches off anabolic processes that consume ATP for the syntheses of fatty acids, triglyceride, cholesterol, and glucose. ${ }^{20)}$ Therefore, AMPK activation in skeletal muscle is associated with lower plasma glucose levels because it increases glucose uptake for fatty acid oxidation by inhibiting fatty acid synthesis by directly phosphorylating acetyl-CoA carboxylase (ACC) and PGCl $\alpha{ }^{20)}$ In the present study, AIII was found to increase glucose metabolism by directly activating $\mathrm{PGC} 1 \alpha$, and thus, activating the AMPK pathway.

Under conditions of energy deficiency, such as, caloric restriction, which increases intracellular $\mathrm{NAD}^{+}$levels and ac- tivates SIRT1 via PGC1 $\alpha$ deacetylation. ${ }^{27)}$ AMPK and SIRT1 regulate each other and target molecules, such as, $\mathrm{PGCl} \alpha$, forkhead box-containing protein, and endothelial NO synthase. ${ }^{25)}$ Previous studies have shown that AMPK activation and PGC1 $\alpha$ phosphorylation precede SIRT1 activation and PGC1 $\alpha$ deacetylation, and in the present study, SIRT1 expression was found to be significantly increased by AIII in $\mathrm{C} 2 \mathrm{C} 12$ myotubes.

Summarizing, our data showed that AIII improves mitochondrial biogenesis and glucose metabolism by activating the PGC1 $\alpha /$ SIRT1/AMPK pathway in skeletal muscle cells Thus, this finding suggests that AII has therapeutic potential for the treatment of obesity and T2DM by improving skeletal muscle energy metabolism.

Acknowledgment This research was supported by the Korean Health Technology R\&D Project through the Korean Health Industry Development Institute (KHIDI) funded by the Ministry of Health \& Welfare, Republic of Korea (Grant number: HI15C0127).

Conflict of Interest The authors declare no conflict of interest.

\section{REFERENCES}

1) Ginsberg HN, MacCallum PR. The obesity, metabolic syndrome, and type 2 diabetes mellitus pandemic: Part I. Increased cardiovascular disease risk and the importance of atherogenic dyslipidemia in persons with the metabolic syndrome and type 2 diabetes mellitus. J. Cardiometab. Syndr., 4, 113-119 (2009).

2) World Health Organization. "Obesity and overweight.": ‘http://www. who.int/mediacentre/factsheets/fs311/en/s, cited 10 September 2016.

3) Medina-Gomez G, Vidal-Puig A. Gateway to the metabolic syndrome. Nat. Med., 11, 602-603 (2005).

4) Tseng Y-H, Cypess AM, Kahn CR. Cellular bioenergetics as a target for obesity therapy. Nat. Rev. Drug Discov., 9, 465-482 (2010).

5) Krssak M, Falk Petersen K, Dresner A, DiPietro L, Vogel SM, Rothman DL, Roden M, Shulman GI. Intramyocellular lipid concentrations are correlated with insulin sensitivity in humans: a H-NMR spectroscopy study. Diabetologia, 42, 113-116 (1999).

6) Greco AV, Mingrone G, Giancaterini A, Manco M, Morroni M, Cinti S, Granzotto M, Vettor R, Camastra S, Ferrannini E. Insulin resistance in morbid obesity reversal with intramyocellular fat depletion. Diabetes, 51, 144-151 (2002).

7) DeFronzo RA, Gunnarsson R, Björkman O, Olsson M, Wahren J. Effects of insulin on peripheral and splanchnic glucose metabolism in noninsulin-dependent (type II) diabetes mellitus. J. Clin. Invest., 76, 149-155 (1985).

8) Cantó C, Auwerx J. PGC-1alpha, SIRT1 and AMPK, an energy sensing network that controls energy expenditure. Curr. Opin. Lipidol., 20, 98-105 (2009).

9) Liang H, Ward WF. PGC-1 $\alpha$ : a key regulator of energy metabolism. Adv. Physiol. Educ., 30, 145-151 (2006).

10) Suwa M, Egashira T, Nakano H, Sasaki H, Kumagai S. Metformin increases the PGC-1 $\alpha$ protein and oxidative enzyme activities possibly via AMPK phosphorylation in skeletal muscle in vivo. J. Appl. Physiol., 101, 1685-1692 (2006).

11) Lagouge M, Argmann C, Gerhart-Hines Z, Meziane H, Lerin C, Daussin F, Messadeq N, Milne J, Lambert P, Elliott P, Geny B, Laakso M, Puigserver P, Auwerx J. Resveratrol improves mitochondrial function and protects against metabolic disease by activating SIRT1 and PGC-1 $\alpha$. Cell, 127, 1109-1122 (2006).

12) Song MY, Kang SY, Oh TW, Kumar RV, Jung HW, Park Y-K. The 
roots of Atractylodes macrocephala Koidzumi enhanced glucose and lipid metabolism in $\mathrm{C} 2 \mathrm{C} 12$ myotubes via mitochondrial regulation. Evid. Based Complement. Alternat. Med., 2015, 643654 (2015).

13) Zhou J, Qu F, Yu Y. Chemical and ecological evaluation of a genuine chinese medicine Atractylodes macrocephala Koidz. Afr. J. Tradit. Complement. Altern. Medicines, 8, 405-411 (2011).

14) Li CQ, He LC, Jin JQ. Atractylenolide I and atractylenolide III inhibit lipopolysaccharide-induced TNF- $\alpha$ and NO production in macrophages. Phytother. Res., 21, 347-353 (2007).

15) Dong H, He L, Huang M, Dong Y. Anti-inflammatory components isolated from Atractylodes macrocephala Koidz. Nat. Prod. Res., 22, 1418-1427 (2008).

16) Wang KT, Chen LG, Wu CH, Chang CC, Wang CC. Gastroprotective activity of atractylenolide III from Atractylodes ovata on ethanol-induced gastric ulcer in vitro and in vivo. J. Pharm. Pharmacol., 62, 381-388 (2010)

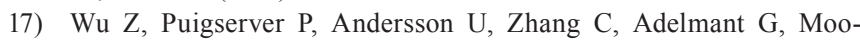
tha V, Troy A, Cinti S, Lowell B, Scarpulla RC, Spiegelman BM. Mechanisms controlling mitochondrial biogenesis and respiration through the thermogenic coactivator PGC-1. Cell, 98, 115-124 (1999).

18) Rohas LM, St-Pierre J, Uldry M, Jäger S, Handschin C, Spiegelman BM. A fundamental system of cellular energy homeostasis regulated by PGC-1 $\alpha$. Proc. Natl. Acad. Sci. U.S.A., 104, 7933-7938 (2007).

19) Widlansky ME, Wang J, Shenouda SM, Hagen TM, Smith AR, Kizhakekuttu TJ, Kluge MA, Weihrauch D, Gutterman DD, Vita JA. Altered mitochondrial membrane potential, mass, and mor- phology in the mononuclear cells of humans with type 2 diabetes. Transl. Res., 156, 15-25 (2010).

20) Lage R, Diéguez C, Vidal-Puig A, López M. AMPK: a metabolic gauge regulating whole-body energy homeostasis. Trends Mol. Med., 14, 539-549 (2008).

21) Zong H, Ren JM, Young LH, Pypaert M, Mu J, Birnbaum MJ, Shulman GI. AMP kinase is required for mitochondrial biogenesis in skeletal muscle in response to chronic energy deprivation. Proc. Natl. Acad. Sci. U.S.A., 99, 15983-15987 (2002).

22) Cantó C, Gerhart-Hines $Z$, Feige JN, Lagouge M, Noriega L, Milne JC, Elliott PJ, Puigserver P, Auwerx J. AMPK regulates energy expenditure by modulating $\mathrm{NAD}^{+}$metabolism and SIRT1 activity. Nature, 458, 1056-1060 (2009).

23) Shan G-S, Zhang L-X, Zhao Q-M, Xiao H-B, Zhuo R-J, Xu G, Jiang H, You X-M, Jia T-Z. Metabolomic study of raw and processed Atractylodes macrocephala Koidz by LC-MS. J. Pharm. Biomed. Anal., 98, 74-84 (2014).

24) Kang T-H, Han N-R, Kim H-M, Jeong H-J. Blockade of IL-6 secretion pathway by the sesquiterpenoid atractylenolide III. $J$. Nat. Prod., 74, 223-227 (2011).

25) Ruderman NB, Xu XJ, Nelson L, Cacicedo JM, Saha AK, Lan F, Ido Y. AMPK and SIRT1: a long-standing partnership? Am. J. Physiol. Endocrinol. Metab., 298, E751-E760 (2010).

26) Scarpulla RC. Transcriptional paradigms in mammalian mitochondrial biogenesis and function. Physiol. Rev., 88, 611-638 (2008).

27) Finkel T, Deng C-X, Mostoslavsky R. Recent progress in the biology and physiology of sirtuins. Nature, 460, 587-591 (2009). 